\title{
Clinical application of 18F-fluorodeoxyglucose positron emission tomography for assessment and evaluation after therapy for malignant hepatic tumor
}

\author{
Masakatsu Tsurusaki $\cdot$ Masahiko Okada • \\ Hiroyuki Kuroda $\cdot$ Mitsuru Matsuki • \\ Kazunari Ishii $\cdot$ Takamichi Murakami
}

Received: 16 November 2012/ Accepted: 4 March 2013/Published online: 26 March 2013

(C) The Author(s) 2013. This article is published with open access at Springerlink.com

\begin{abstract}
Positron emission tomography (PET) is widely available and its application with $2-\left[{ }^{18} \mathrm{~F}\right]$ fluoro-2-deoxy-Dglucose $\left({ }^{18} \mathrm{~F}-\mathrm{FDG}\right)$ in oncology has become one of the standard imaging modalities in diagnosing and staging of tumors, and monitoring the therapeutic efficacy in hepatic malignancies. Recently, investigators have measured glucose utilization in liver tumors using ${ }^{18} \mathrm{~F}-\mathrm{FDG}$ and positron emission tomography/computer tomography (PET/CT) in order to establish a diagnosis of tumors, assess their biologic characteristics and predict therapeutic effects on hepatic malignancies. The PET/CT with ${ }^{18}$ F-FDG may further enhance the hepatic malignancy diagnostic algorithm by accurate diagnosis, staging, restaging and evaluating its biological characteristics, which can benefit the patients suffering from primary and metastatic hepatic tumors such as hepatocellular carcinoma (HCC), cholangiocarcinoma (CCC), and metastatic liver tumor.
\end{abstract}

Keywords Positron emission tomography - Liver . Malignant hepatic tumor - Hepatocellular carcinoma . Tumor response

\footnotetext{
M. Tsurusaki $(\bowtie) \cdot$ M. Okada · M. Matsuki · K. Ishii ·

T. Murakami

Department of Diagnostic Radiology, Kinki University,

School of Medicine, 377-2 Ohnohigashi,

Osaka-sayama, Osaka 589-8511, Japan

e-mail: mtsuru@dk2.so-net.ne.jp

H. Kuroda

Department of Radiology,

Izumo Medical Center Hospital, Izumo, Japan
}

\section{Introduction}

Positron emission tomography (PET) is a functional imaging modality that uses positron emitters, such as fluorine-18. Fluorine-18 has a physical half-life of $110 \mathrm{~min}$ and can be synthesized with a cyclotron or delivered as a radiopharmaceuticals from a radiopharmaceutical company to an institute without cyclotron units. While PET has been used for several decades in research tools, its clinical use has grown substantially in the past decade. The most commonly clinically used radiotracer is $2-\left[{ }^{18} \mathrm{~F}\right]$ fluoro-2deoxy-D-glucose (FDG). The FDG-PET has been widely used not only for detecting and staging malignant tumors but also for monitoring therapy response and differentiating malignant from benign lesions. In this review, we would like to show clinical application of FDG-PET for The assessment of malignant hepatic tumors.

\section{Metabolic mechanism of FDG in the liver}

Tumor imaging using FDG is based on the principle of increased glucose metabolism of cancer cells. Like glucose, FDG is taken up by cancer cells via facilitative glucose transporters (Gluts). Gluts are glycoproteins, and so far, 12 isoforms have been identified in different organs. Normal hepatocytes express Glut2, Glut9, and Glut10 [1]. The expression of Gluts, predominantly Glut 1 and Glut 3 , is significantly higher in many cancer cells than in normal cells. Once in the cell, glucose or FDG is phosphorylated by hexokinase to glucose-6-phosphate or FDG-6-phosphate, respectively. The expression of hexokinase and its affinity for/function of phosphorylation of glucose or FDG is often higher in cancer cells than in normal cells; hexokinase II is predominantly expressed in cancer cells. 
Glucose-6-phosphate travels further down the glycolytic or oxidative pathways to be metabolized, unlike FDG-6phosphate, which cannot be metabolized. In normal cells, glucose-6-phospate or FDG-6-phosphate can undergo dephosphorylation and can exit the cells. In many cancer cells, however, the expression of glucose-6-phosphatase is often significantly low; therefore, glucose-6-phospate or FDG-6-phosphate are only minimally dephosphorylated and remain within the cell. On the other hand, because FDG-6-phosphate cannot be metabolized, it is trapped in cells as a polar metabolite and can be visualized by PET (Fig. 1). In normal liver parenchyma, the concentration of glucose-6-phosphatase is high, which causes rapid clearance of FDG from the liver. This may account for the mild intensity of the normal liver on whole-body PET, especially at later imaging times post tracer injection [2]. In fact, in many publications and clinical routines, the PET intensity of the liver has often been used as a reference for background uptake.

\section{Hepatocellular carcinoma}

Hepatocellular carcinoma (HCC) or hepatoma develops via malignant transformation of hepatocytes and is common in the settings of chronic liver changes or cirrhosis. The HCC is the most common primary malignancy of the liver, accounting for about $80 \%$ of malignant neoplasms of the liver [3]. The diagnosis is based on screening risk populations with measurements of tumor marker, such as serum alpha fetoprotein or PIVKA II, and liver ultrasound. MRI, CT, and/or lipiodol angiography with follow-up CT are used in inconclusive cases to establish the diagnosis [4].

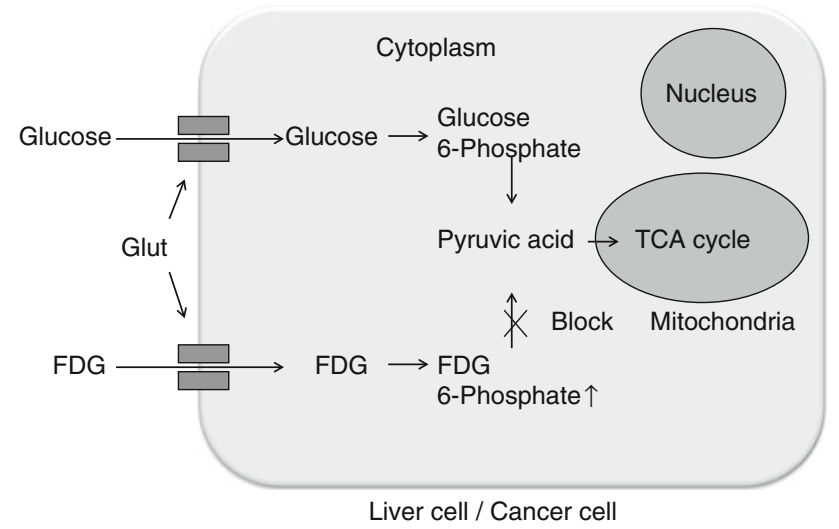

Fig. 1 Uptake of FDG. FDG is a glucose analog that is taken up by metabolically active cells by means of facilitated transport via glucose transporters (Glut) in the cell membrane. In the cell cytoplasm, FDG undergoes phosphorylation to form FDG-6-phosphate (6P), which, unlike glucose, cannot undergo further metabolism and becomes trapped within the cell
Biopsy is only performed on patients in whom the radiological diagnosis cannot be made [5].

Facilitative Gluts do not appear to be overexpressed in $\mathrm{HCC}$ as often as in other malignant tumors. Zimmerman et al., and Roh et al. [6, 7] reported the expression of Glut1 in 2 of 35 and 1 of 22 examined HCC cases, respectively. Delbeke et al. [8] examined a series of 23 patients with HCC. During visual assessment, the tumor-to-liver ratio was definitely high in 13 patients, equivocal (slightly increased compared with that of livers) in three patients, and poor (same or less than that of normal livers) in seven patients. The sensitivity of FDG-PET for HCC is approximately $50 \%$ [8-10]. There appears to be some association between the histological differentiation of HCC and FDG uptake, with poorly differentiated tumors showing higher intensity on FDG-PET, which may be explained by the enzymology of HCC (Fig. 2). The concentration of glucose-6-phosphatase is high in normal livers, which causes rapid clearance of glucose-6-phospate or FDG-6-phosphate from hepatocytes, with consequent mild appearance of the liver on PET. The enzymology of well-differentiated HCC resembles that of the normal liver, which may explain the mild FDG uptake or nonvisualization of these tumors on PET (Fig. 3). Moderately to poorly differentiated HCC tumors have lower levels of glucose-6-phosphatase and higher levels of hexokinase, which probably causes intense FDG uptake of these tumors on PET [2, 8, 11, 12]. In addition, Trojan et al. [13] described that there appears to be some association between FDG uptake and tumor-volume doubling time as well as between FDG uptake and tumor size in a series of 14 HCC tumors. Therefore, FDGPET could possibly be used to assess the effect of treatment in larger and less-differentiated HCC. They also described the sensitivity of FDG-PET for the imaging of HCC is approximately $50 \%$. Nevertheless, in patients with moderately or poorly differentiated $\mathrm{HCC}$, the sensitivity was $88 \%$, which may contribute to an effective noninvasive staging. Shiomi et al., and Kong et al. [14, 15] demonstrated the usefulness of FDG-PET in predicting the outcome in patients with HCC.

Detection of extrahepatic FDG-avid metastases originating from $\mathrm{HCC}$ has also been reported; especially in cases of less-differentiated HCC, metastases appear to be more FDG avid [2, 13, 56]. Sugiyama et al., reported that the sensitivity of FDG-PET was $83 \%$ for extrahepatic metastases larger than $1 \mathrm{~cm}$ in greatest diameter and $13 \%$ for lesions less than or equal to $1 \mathrm{~cm}$. There were no falsepositive lesions in all lesions [56]. On the other hand, another report revealed that the accuracy of chest CT was significantly superior compared with the accuracy of PET imaging for detecting lung metastases. The detection rate of metastatic pulmonary nodules $\geq 1 \mathrm{~cm}$ was $92.3 \%$, when $<1 \mathrm{~cm}$ was $20 \%$ in PET imaging [57]. 

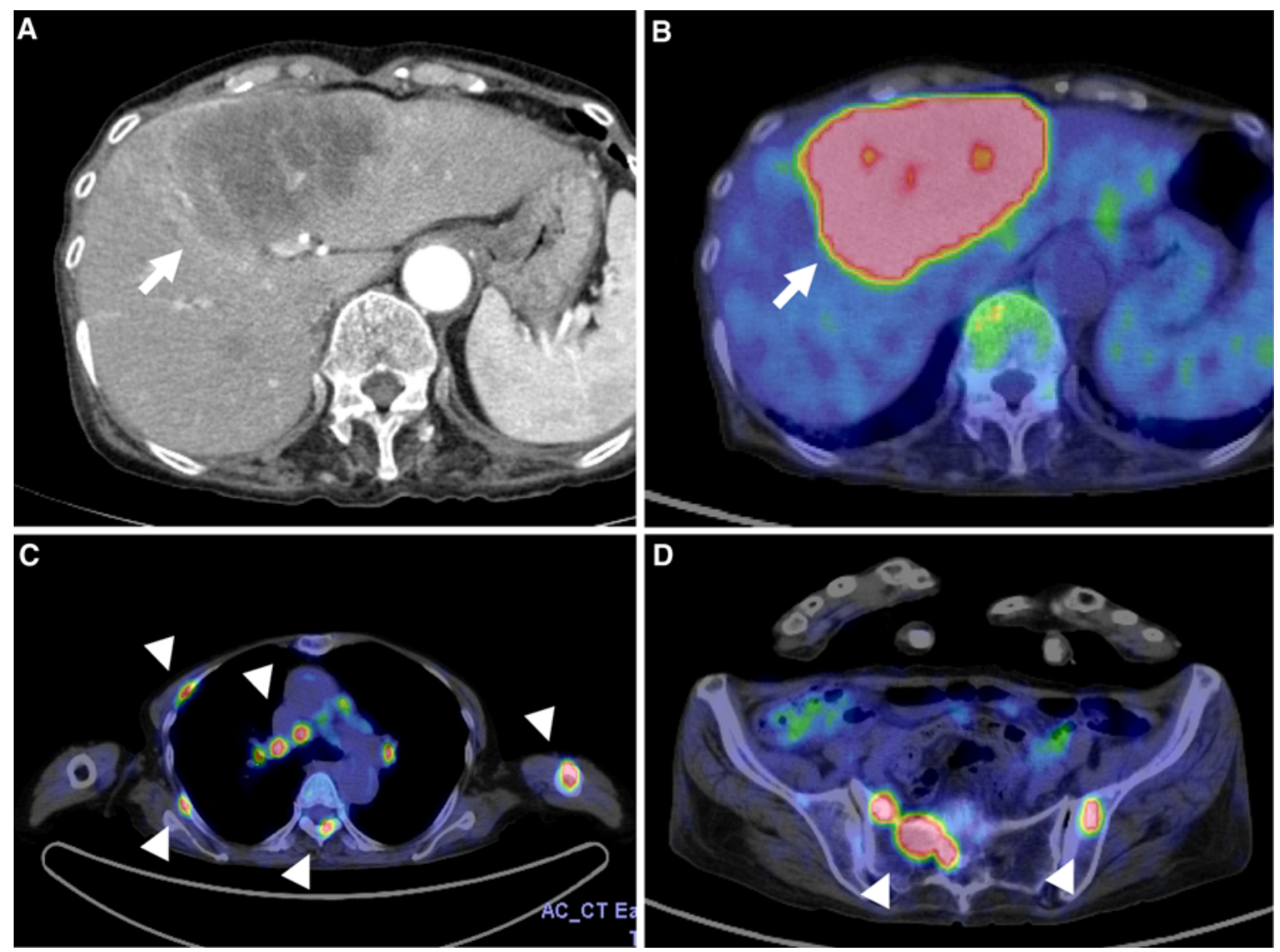

Fig. 2 A 83-year-old woman who had HCC that detected a huge hepatic mass by contrast-enhanced CT (a, arrow). PET markedly revealed FDG avidity of the hepatic mass (b, arrow) as well as FDG

Although more data are needed to establish the clinical role of FDG-PET in HCC, in our experience, it is very helpful for assessment of the malignant potential of hepatic lesions of unknown origin through simultaneous visualization of the liver and extrahepatic tissue and for that of known HCC with clinically suspected extrahepatic metastasis (Figs. 2, 4). Nonetheless, a negative FDG-PET scan in patients with a solitary hepatic lesion does not exclude the possibility of HCC.

\section{Metastatic liver tumor}

Metastatic disease accounts for the majority of malignant lesions in the liver. Often, the presence of liver metastases is the main determinant of survival and guides the therapeutic strategy, particularly in patients with colorectal cancer $[16,17]$.

Zimmerman et al. [6] studied the expression of Glut1 in hepatic metastases originating from different primaries and reported that Glut1 was overexpressed in hepatic metastases from 3 of 5 lung primaries, 7 of 11 pancreatic

avid deposits along multiple bones (ribs, vertebra, humerus, and pelvis) and mediastinal lymph nodes (c, d, arrow head). Core-needle biopsy of liver mass indicated undifferentiated HCC

primaries, 7 of 9 colon primaries, 2 of 7 breast primaries, 2 of 2 squamous cell primaries, 1 of 3 biliary tract primaries, and none of the neuroendocrine primaries that were examined. To our knowledge, expression of other Gluts, such as Glut3, in hepatic metastases has not previously been reported.

The FDG-PET has been proven to be highly sensitive in detecting hepatic metastases from different primaries. Delbeke et al. [8] studied the diagnostic value of FDG-PET in hepatic metastases measuring $1 \mathrm{~cm}$ and more and detected all 66 metastatic lesions originating from various primaries, such as the colon, pancreas, esophagus, sarcoma, and parotid. Similar results that showed the overall greater sensitivity of PET, compared with that of spiral computed tomography (CT), have been reported by other groups, particularly for CT findings that were indeterminate $[11,12,18]$. In cases of known solitary hepatic metastasis diagnosed using CT, several groups have reported the discovery of additional hepatic metastases using FDG-PET [19-21]. This is of particular importance in preoperative evaluation of solitary hepatic metastasis because detection of additional lesions often changes the management. Retrospective data 

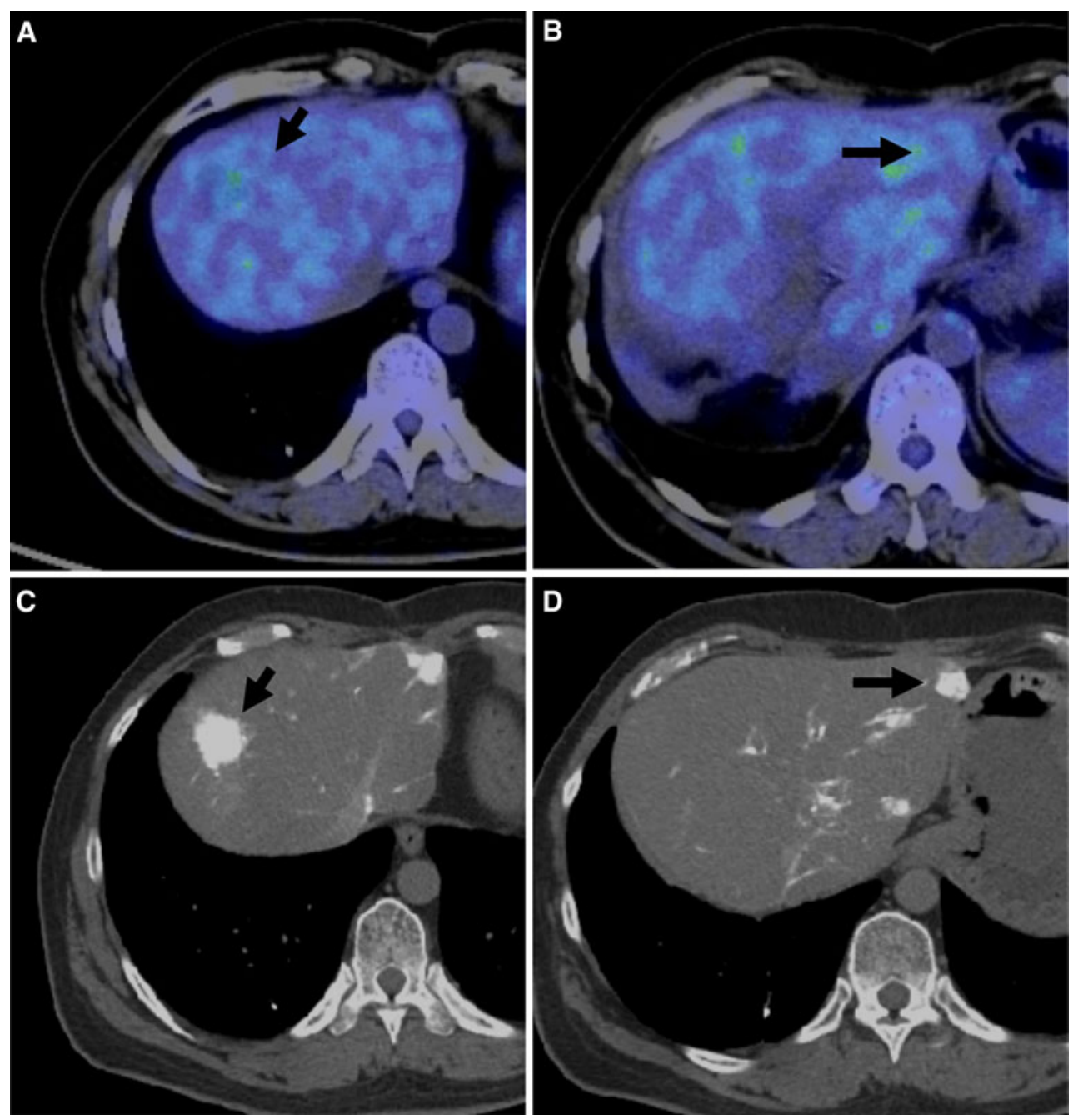

Fig. 3 A 38-year-old man who had HCC resection 4 years before had an intrahepatic HCC recurrence and received transarterial infusion therapy. No metabolically active lesion compared with background of

the liver was detected by PET/CT fused images (a, b, arrows). CT hepatic arteriography showed multiple hypervascular nodules in each lobe of the liver (c, d, arrows)

by Fernandez et al., have shown that using FDG-PET to assess patients with colon cancer liver metastases considered for partial hepatectomy was associated with long-term survival. The survival of such patients was superior to that of patients with the same condition for whom only standard anatomical imaging methods were used in the selection of surgery. Presumably, PET was used to select those patients who did not have extrahepatic metastases and thus, were most likely to benefit from partial hepatectomy [22]. Additionally, in cases of suspected recurrent colorectal cancer, FDG-PET is more sensitive than CT for discovering hepatic metastases and has the potential of detecting disease earlier than CT when metastatic disease is more amenable to curative resection [21, 23]. The FDG-PET should be especially considered in settings of increased carcinoembryonic antigen levels to assess hepatic metastases because it has proven to be more sensitive than CT for this purpose [25].

Yang et al., reviewed PET and magnetic resonance imaging (MRI) studies of 30 patients with histopathologically proven $(n=27)$ or clinically suspected $(n=3)$ hepatic metastases from non-hepatic primaries. The sensitivity, specificity, positive predictive values, and negative predictive values on MRI were $85.7,100,100$, and $89 \%$, respectively, compared with $71,93.7,90.9$, and $79 \%$, respectively, on PET. The differences in the results between the two methods were not statistically significant [24]. Bohm et al. [11] demonstrated similar results.

Besides, another meta-analysis of the literature on detection of hepatic metastases from colorectal, gastric, and esophageal cancers using ultrasonography (US), CT, MRI, and PET found that in studies with a specificity higher than $85 \%$, the mean weighted sensitivity was $55 \%$ $(95 \%$ CI 41,68$)$ for US, $72 \%(95 \%$ CI 63,80$)$ for CT, $76 \%(95 \%$ CI 57, 91) for MRI, and $90 \%$ (95\% CI 80, 

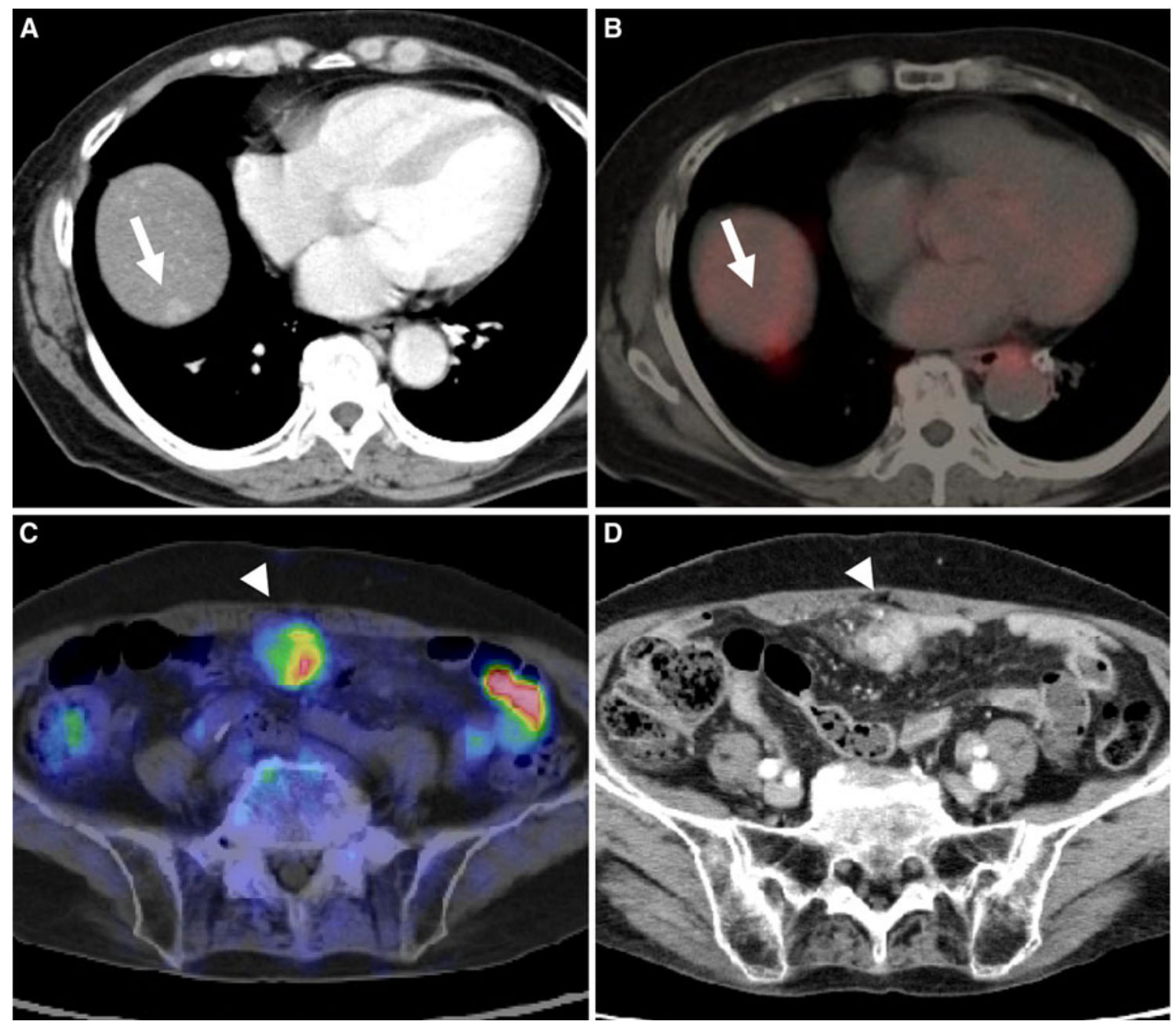

Fig. 4 A 58-year-old woman with hepatitis $\mathrm{C}$ infection who had been detected as having a right hepatic nodule on contrast-enhanced CT (a, arrow). PET was requested for further assessment, and revealed FDG avidity of the hepatic mass (b, arrow) as well as FDG avid deposits below the anterior abdominal wall (c, arrow head). Contrast-

97) for PET. The conclusion was that at equivalent specificity, PET is the most sensitive noninvasive imaging modality for diagnosing hepatic metastases from colorectal, gastric, and esophageal cancers [25]. The CT can achieve higher sensitivity, but at the expense of specificity. In our experience, PET is of particular benefit in cases of indeterminate CT findings (Fig. 5). This was shown by Marom et al. [26] in metastatic lung cancer, where in a prospective study of 100 patients, nearly twice as many lesions in the liver were identified using CT than PET; however, all of the incremental lesions identified on CT were false positives.

False-negative PET results for hepatic metastases due to the lower image resolution of PET compared with that of spiral CT and MRI has been reported [11, 27, 28]. It should be considered, however, that these publications were based

enhanced CT showed hypervascular mass in the peritoneum at the same portion (d, arrow head). Image characteristics of the left hepatic mass on CT and Alpha Fetoprotein level of $897 \mathrm{ng} / \mathrm{ml}$ indicated HCC as the primary, and the peritoneal mass seems to be extrahepatic metastasis

on non-attenuation corrected images, which may have had lower sensitivity, especially deeper in the abdomen because the deeper areas of the abdomen generally appear much fainter on non-attenuation corrected than on attenuationcorrected PET images. Generally, the role of PET in detecting sub centimeter lesions should be redefined, considering the sophisticated image correction, reconstruction algorithms, and higher image resolution of current PET machines. Nonetheless, detection is limited by the sensitivity and resolution of the scanner as well as the background tissue radioactivity levels in the normal tissue. Further improvements in image resolution from the current $1-\mathrm{cm}$ reconstructed resolution to a few millimeters can be expected with the development of small surface area crystal elements in combination with alternative positionsensitive photomultiplier tubes and the implementation of 


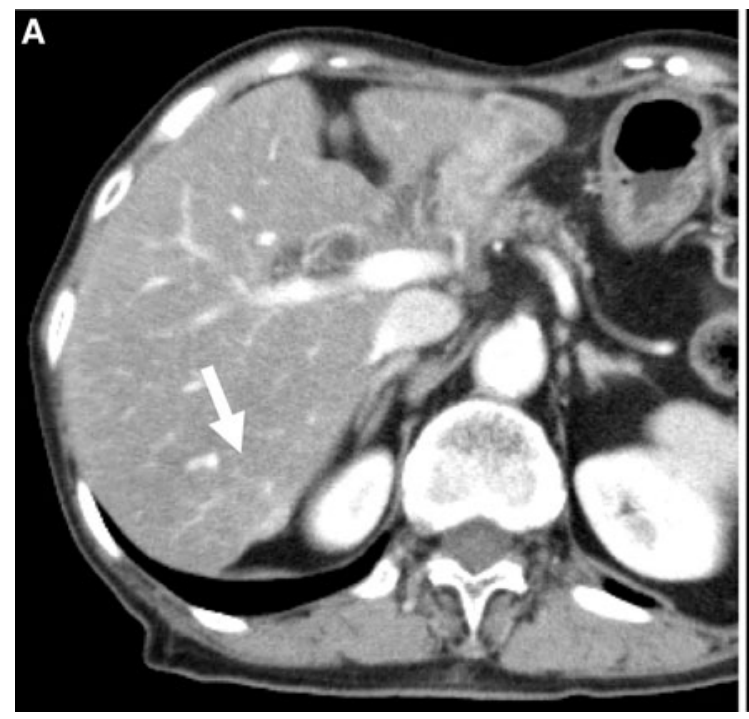

Fig. 5 A 73-year-old man who underwent sigmoidectomy for colon cancer 2 years ago. Contrast-enhanced CT could not clearly indicate a solid lesion in segment VI of the liver (a,arrow). PET was requested

depth of interaction measurements $[29,30]$. However, it is still rare for the reconstructed scanner resolution in patient imaging to match the optimal resolution of the scanner because there often are not enough photon events to depict the true resolution. Thus, at present, the structural resolution of anatomical imaging remains superior to that of PET. Nonetheless, the diagnostic accuracy of PET is generally superior to that of anatomical imaging because of its physiological basis for lesion detection. Another approach to increase detectability of liver metastases is through acquisition of dynamic PET and the formation of parametric images of the influx constant as described by Zasadny and Wahl [31]; this approach increases the tumorto-background signal ratio and potentially may improve detectability of small lesions, but requires more time than standard imaging. False-negative FDG-PET findings due to underestimation of uptake, misregistration of foci, and recent completion of chemotherapy has also been reported $[32,33]$. The latter is likely associated with microscopic remnant disease at the completion of chemotherapy that grows and increases in volume, with subsequent visualization on PET once chemotherapy has been terminated. However, no information is available in the literature on the time interval after completion of chemotherapy during which PET can give false negative results. On basis of the authors' experience, this time interval is about 4-6 weeks.

Underestimation of the uptake of malignant lesions that cause false-negative findings on PET can occur because of physiological movements of the liver during emission scans. The liver is an upper abdominal organ that moves with respiratory movement of the diaphragm. Emission

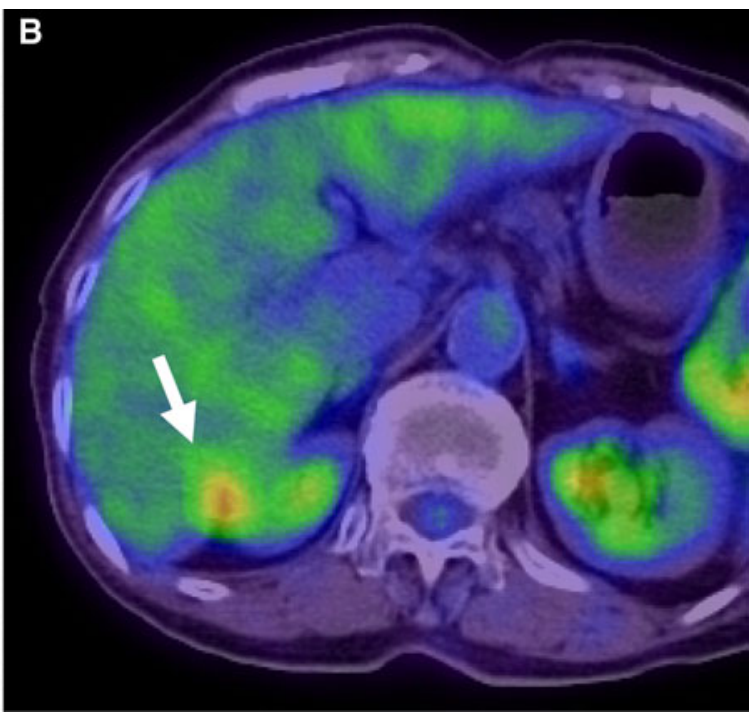

for further assessment, and revealed FDG avidity at the same portion in the segment VI of the liver (b, arrow). Surgical specimen indicated metastatic adenocarcinoma

scans are acquired over several minutes during which hepatic lesions, especially those at the dome, are in a repetitive craniocaudal pendulous movement. The respiratory excursion of the liver has been estimated to be $10-25 \mathrm{~mm}[34,35]$. Therefore, it is conceivable that cranial and caudal portions of small lesions are registered only for half of the acquisition time, and hence, their uptake is underestimated so that they appear less intense on images than they really are. The degree of this underestimation is variable, and particularly in the case of a subcentimeter lesion, this may even lead to non-visualization of the lesion, as reported by Rohren et al. [32]. One way to overcome this problem is to increase the target-to-background count ratio by increasing the acquisition time. This can be done by increasing the acquisition time of the middle and upper abdomen emission frames while acquiring the whole-body scan, if possible, on the PET machine. A different approach to solve this problem would be respiratory gating, in which emission data collected during certain phases of the respiratory cycle are used for image reconstruction. This approach results in better visualization of small lesions with the disadvantage of longer acquisition time [30, 36].

False-positive findings for malignancy on PET due to intrahepatic abscess; penetrating gallbladder empyema; or benign inflammatory lesions, such as regenerative nodules in a cirrhotic liver, have also been reported [8, 11, 19].

Given the higher sensitivity of PET in detecting hepatic and especially extrahepatic metastasis, it is conceivable that PET will be increasingly employed for preoperative staging of malignant tumors. 


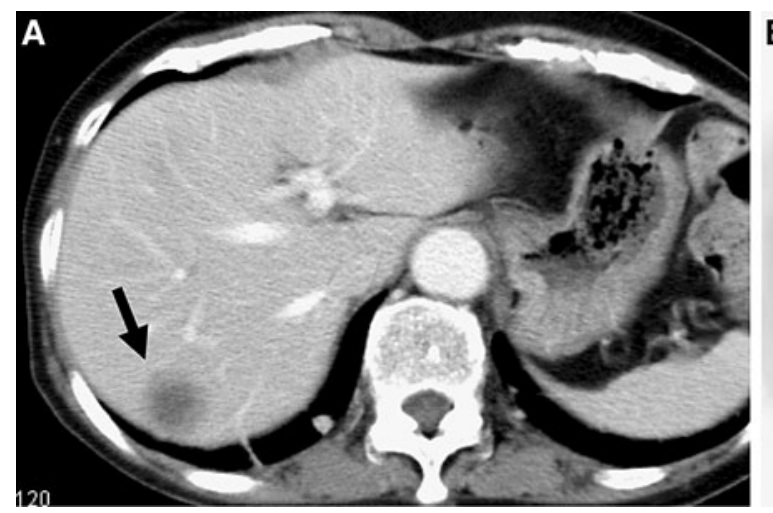

Fig. 6 A 55-year old man who had been detected pathological proven CCC in the posterior right hepatic lobe. Contrast-enhanced CT shows minimal enhancement with unclear margin (a, arrow) and PET reveals hypermetabolism (b, arrow)

\section{Cholangiocellular carcinoma}

Cholangiocarcinoma (CCC) originates from the epithelial cells of the biliary tract. Next to HCC, it is the second most common primary tumor of the liver, accounting for about $5-30 \%$ of primary hepatic malignancies [3]. Biliary obstruction with jaundice is the most common clinical feature in hilar CCC, whereas it is uncommon in peripheral $\mathrm{CCC}$. The diagnosis of $\mathrm{CCC}$ has been based on the clinical picture, laboratory data, radiological imaging, and histology, although the latter is often inconclusive in differentiating CCC from metastatic adenocarcinoma. Currently, workup generally consists of MRI including magnetic resonance cholangiopancreatography (MRCP), CT, endoscopic retrograde cholangiopancreatography (ERCP), and percutaneous transhepatic cholangiography (PTC). Overall, the prognosis of this tumor is dismal, with a 5-year survival as low as $17 \%$; however, improved survival of 22-32\% at 5 years has been reported with portal or arterial embolization followed by trisegmentectomy [37, 38]. Therefore, preoperative assessment for hepatic and extrahepatic metastases probably has prognostic value.

Glut1 is not expressed in normal bile ducts but has been described to be strongly expressed in CCC [7, 39]. Overall, CCC appears to be highly FDG avid and can be visualized on PET if sufficient tumor volume is present (Fig. 6). Delbeke et al. [8] evaluated eight patients with CCC, and all lesions demonstrated intense FDG uptake. Hilar and extrahepatic $\mathrm{CCC}$, however, have been reported to be less intense on FDG-PET than on the peripheral CCC, which may be associated with the smaller size and/or higher mucin content of the hilar tumors than those of the peripheral ones [40-42]. Peripheral CCC accounts for about $10 \%$ of all CCC and often has a characteristic central photopenia on FDG-PET, which corresponds to the central core of fibrotic tissue and desmoplastic reaction provoked by the neoplastic cells; on contrast-enhanced CT or MRI, this is evident by early moderate peripheral enhancement followed by progressive and concentric filling $[42,43]$. In assessing the ability of FDG PET to detect and diagnose CCC, in a prospective study, Kim et al. [58] found overall values for sensitivity, specificity, PPV, NPV, and accuracy of FDG PET/CT in primary tumor detection were $84.0,79.3,92.9,60.5$, and $82.9 \%$, respectively. Otherwise, a recent study investigating FDG PET/CT by Jadvar et al. [59] found sensitivity and specificity to be 94 and $100 \%$, respectively. These results lead to the appearance of not so many false-positive cases, therefore, it may not so important that the influence of secondary changes by CCC such as bile duct obstruction or infection. Kato et al., reported $100 \%$ specificity for regional nodal involvement on FDG-PET [39]. Especially in cases of peripheral CCC, PET should be considered to evaluate patients for extrahepatic metastases; peripheral CCC usually attains a large size before it becomes clinically apparent because it does not obstruct the central biliary system.

\section{Evaluation of response after local and systemic treatment}

For selective treatment of liver tumors, techniques such as radiofrequency ablation (RFA), transcatheter chemoembolization, and arterial chemotherapy infusion have been increasingly used. Despite improvements, these techniques are hampered by limitations in monitoring the effect of treatment. Especially, because the rate of residual disease in tumors larger than $3 \mathrm{~cm}$ appears to be as high as $48 \%$, short-term follow-up and repeat of the local ablation would be of great benefit. Incomplete ablation because of the close proximity of a tumor to major vessels and the resulting so-called heat-sink effect has been reported as well $[44,45]$. 

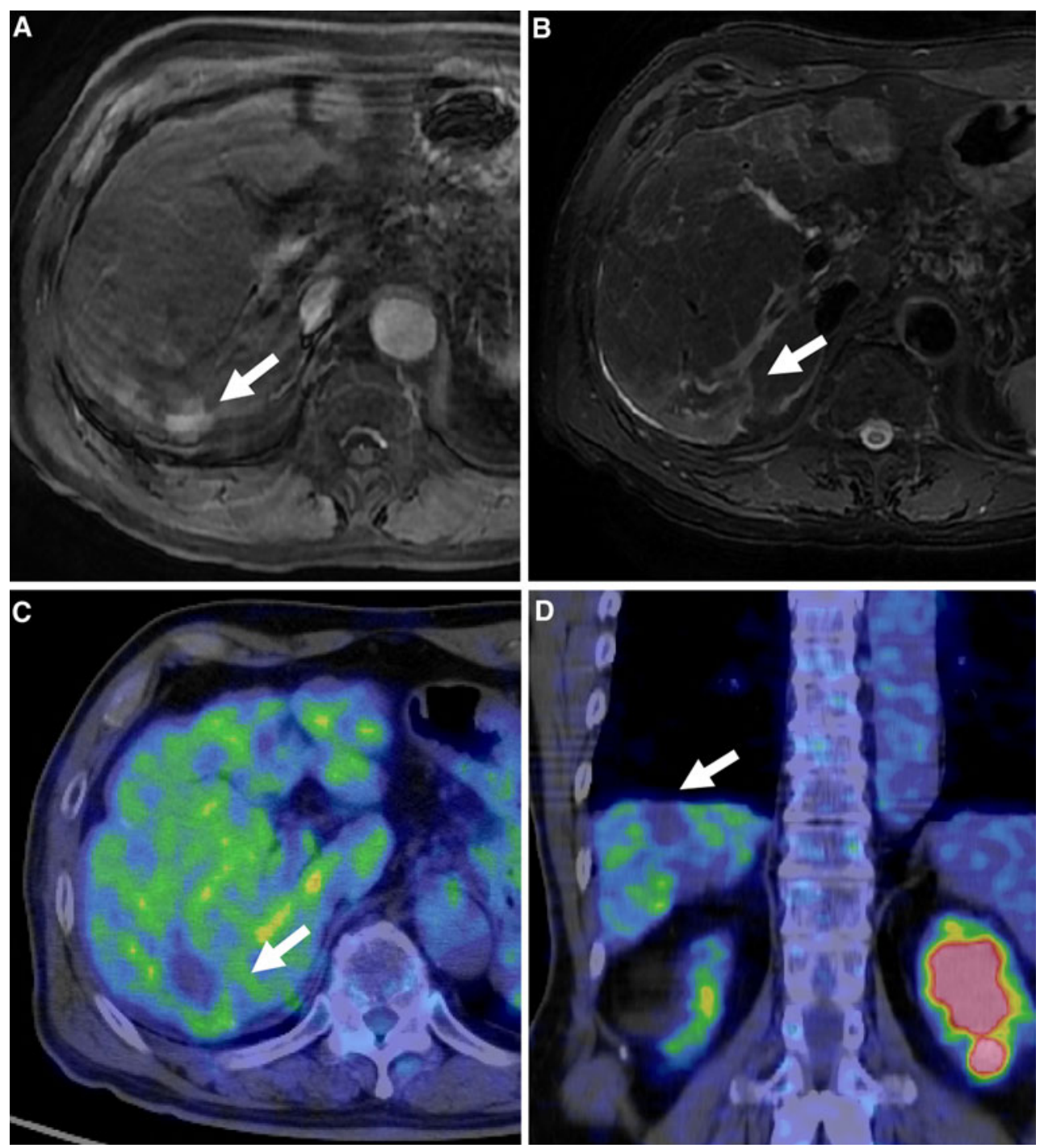

Fig. 7 A 61-year-old man with $\mathrm{HCC}$ in the posterior right hepatic lobe, who underwent RFA. Thirty days after RFA, gadoxetic acidenhanced MRI indicated contrast-enhancement in early phase surrounding the ablation site (a, arrow) with high signal on T2 weighted image (b,arrow). It was diagnosed that there was viable residual tumor on MRI. However, PET indicated photopenia in that

In the first month after RFA, the ability of CT and MRI to detect residual tumors is limited because of the presence of ablation-induced necrosis, edema, and hyperemia in and around the ablated lesion. Therefore, CT or MRI at 1 month are typically performed to assess for residual tumors [44, 45]. There has been increasing evidence that PET is capable of detecting residual tumors earlier than CT and MRI. So far, it has been documented that PET is capable of visualizing residual disease as early as 7 days after RFA. Donckier et al., compared PET and CT in 28 metastatic liver lesions 1 week, 1 month, and 3 months after RFA. In all 28 lesions, CT scans at all time points revealed large nonenhancing regions at the sites of ablation, without indication

location without evidence for residual uptake consistent with complete ablation (c, d, arrow). Six months later, there was still complete photopenia indicating no presence of viable residual tumor. It confirmed the correct assessment of differential diagnosis between residual tumor recurrence and false positive lesion after ablation by PET

of residual tumors. The PET performed 1 week after RFA, however, detected residual disease in four lesions, which was confirmed by either histology $(n=3)$ or $\mathrm{CT}$ at 6 months $(n=1)$. In the remaining 24 lesions, complete ablation was visualized on PET at 1 week as total photopenia, which was subsequently confirmed on follow-up CT or PET with a median follow-up time of 11 months [46]. Again, this is based on the simple fact that cell death is followed by an immediate decrease in FDG uptake of the tumor mass on PET (Fig. 7).

Besides, the multi-targeted tyrosine kinase inhibitor sorafenib was studied in HCC on the basis of tumor responses and stable disease seen during the early development of the 
drug [47]. Sorafenib can be considered standard of care for patients with advanced and metastatic HCC who are not candidates for curative or locoregional therapies such as TACE for the results of SHARP study [48]. Some investigators have reported that FDG uptake monitored tumor response to sorafenib in HCC, or the degree of FDG uptake, is an independent prognostic factor in patients with HCC who undergo sorafenib treatment in patients with positive FDGPET scans at baseline [49, 50], however, there are not so many investigations. Therefore, it is still controversial what is the role of monitoring response to sorafenib in HCC. Compared with HCC, there are many previous reports that the evaluation of response for the systemic treatment with hepatic colorectal metastasis, particularly in anti-cancer molecular agents such as bevacitumab that is an antiangiogenic agent causing normalization of the tumor microvasculature, potentiates the effect of cytotoxic agents on colorectal liver metastases. De Bruyne et al., reported that some parameters of FDG-PET were better predictors for bevacizumab for colorectal liver metastases [51], in addition, other reports concluded early changes in PET/CT seem to be predictive of longer progression free survival in the patients with FOLFOX6 and bevacizumab in non-optimally resectable liver metastases from colorectal cancer [52]. In the future, it seems to be expected that early monitoring of response to treatment is one of the cornerstones of personalized treatment for the liver metastasis from more additional results of prospective studies. The SUV has been considered by some to be a useful tool for differentiating between malignant and benign etiologies of FDG foci $[9,10]$. However, this view remains controversial. Recently, Israel et al. [11] found that there was no statistical difference between the SUV of premalignant, malignant, benign, and physiologic lesions in an evaluation of unexpected gastrointestinal foci of FDG detected by PET/CT.

Although widely available and convenient to use, standardized uptake value (SUV) measurements can be influenced by a variety of biologic and technologic factors such as scanner, reconstruction parameters, serum glucose, and other some factors. Scanner and reconstruction parameters can significantly affect SUV measurements. When using serial SUV measurements to assess early response to therapy, imaging should be performed on the same scanner using the same image acquisition and reconstruction protocols. In addition, attention to detail is required for accurate determination of the administered radiopharmaceutical dose. Although widely available for SUV measurements, Seo et al., reported the tumor to nontumor SUV ratio (TNR) was more useful than SUV that was calculated as follows: TNR $=$ tumor SUV/nontumor SUV, where the nontumor SUV was defined as the average of SUVs at five points in nontumor liver tissues. They also described that the overall and disease-free survival rates in the high TNR $(\geq 2.0)$ group were significantly lower than in the low TNR
$(<2.0)$ group. In multivariate analysis, a high $\alpha$-fetoprotein level (risk ratio, 5.46; $P=0.003$; risk ratio, 8.78; $P=0.006$ ) and high TNR (risk ratio, $1.3 ; P=0.03$; risk ratio, 1.6; $P=0.02$ ) were independent predictors of postoperative recurrence and overall survival in theirs HCC cases [53].

Some previous reports appear to show that acute hepatitis such as radiation-induced hepatitis or acute on chronic hepatitis caused by viral infection or drug can be a potential cause of false-positive findings of malignancy on FDG PET scans, and PET images should carefully be compared with the distribution change of hepatitis.

\section{Future prospect}

The FDG-PET has been shown to have limited sensitivity for the detection of some HCC tumors because of their variable FDG uptake, 11C-acetate-PET has been used to complement FDG-PET in a dual-tracer PET scan. Ho et al. [54] found that well-differentiated HCCs preferentially accumulate 11C-acetate, whereas poorly differentiated tumors tend to preferentially accumulate FDG. Delbeke et al. [55] suggest that different uptake or tracers by lesions can narrow down a differential diagnosis. On the basis of tracer avidity to different types of HCC lesions, dual-tracer PET (11C-acetate and FDG) could lead to increased sensitivity in detecting all HCC. Ho et al. [53] found that none of $23 \mathrm{HCC}$ lesions in their study population had $100 \%$ sensitivity using both tracers.

Recently, MR imaging with a liver specific contrast agent such as Gd-EOB-DTPA, is one of the most useful for detection and characterization of hepatic tumors. New PET equipment such as PET/MRI scanners are now available in selected departments also in Japan, however, there are few reports that evaluate the clinical values of the liver using PET/MRI scanner. Future studies will show whether hybrid PET/MRI is of greater clinical value than PET/CT and retrospective image fusion techniques.

\section{Conclusion}

The FDG-PET imaging has an important role in determining if metastases are present in the liver and also whether the disease has spread beyond the liver. Such information is critical for planning surgical resections of liver metastases. While FDG-PET can fail to detect many HCCs, it does detect many of the moderately to poorly differentiated ones, and other PET tracers are showing promise for detecting better differentiated HCCs. While low-volume CCCs can escape detection on FDG-PET, 
higher volume lesions are well detected. Thus, with increasingly broad indications for FDG-PET imaging, it is expected that FDG-PET (and PET/CT) of the liver will play a bigger and increasingly important role in detecting and monitoring the treatment of tumors of the liver.

Conflict of interest There is no financial support or any relationships that pose conflict of interest.

Open Access This article is distributed under the terms of the Creative Commons Attribution Noncommercial License which permits any noncommercial use, distribution, and reproduction in any medium, provided the original author(s) and the source are credited.

\section{References}

1. Joost HG, Thorens B. The extended GLUT-family of sugar/polyol transport facilitators: nomenclature, sequence characteristics, and potential function of its novel members. Mol Membr Biol. 2001;18(4):247-56.

2. Ho CL, Yu SC, Yeung DW. 11C-acetate PET imaging in hepatocellular carcinoma and other liver masses. J Nucl Med. 2003; 44(2):213-21.

3. Levy AD. Malignant liver tumors. Clin Liver Dis. 2002;6(1): 147-64.

4. Bruix J, Sherman M; Practice Guidelines Committee; American Association for the Study of Liver Diseases. Management of hepatocellular carcinoma. Hepatology. 2005;42(5):1208-36.

5. Ryder SD; British Society of Gastroenterology. Guidelines for the diagnosis and treatment of hepatocellular carcinoma (HCC) in adults. Gut. 2003;52 Suppl 3:iii1-8.

6. Zimmerman RL, Burke M, Young NA, Solomides CC, Bibbo M. Diagnostic utility of Glut-1 and CA 15-3 in discriminating adenocarcinoma from hepatocellular carcinoma in liver tumors biopsied by fine-needle aspiration. Cancer. 2002;25;96(1):53-7.

7. Roh MS, Jeong JS, Kim YH, Kim MC, Hong SH. Diagnostic utility of GLUT1 in the differential diagnosis of liver carcinomas. Hepatogastroenterology. 2004;51(59):1315-8.

8. Delbeke D, Martin WH, Sandler MP, Chapman WC, Wright JK Jr, Pinson CW. Evaluation of benign vs malignant hepatic lesions with positron emission tomography. Arch Surg. 1998;133(5): $510-5$.

9. Iwata Y, Shiomi S, Sasaki N, Jomura H, Nishiguchi S, Seki S, Kawabe J, Ochi H. Clinical usefulness of positron emission tomography with fluorine-18-fluorodeoxyglucose in the diagnosis of liver tumors. Ann Nucl Med. 2000;14(2):121-6.

10. Schroder O, Trojan J, Zeuzem S, Baum RP. Limited value of fluorine-18-fluorodeoxyglucose PET for the differential diagnosis of focal liver lesions in patients with chronic hepatitis $\mathrm{C}$ virus infection. Nuklearmedizin. 1998;37(8):279-85.

11. Bohm B, Voth M, Geoghegan J, Hellfritzsch H, Petrovich A, Scheele J, Gottschild D. Impact of positron emission tomography on strategy in liver resection for primary and secondary liver tumors. J Cancer Res Clin Oncol. 2004;130(5):266-72.

12. Vitola JV, Delbeke D, Sandler MP, Campbell MG, Powers TA, Wright JK, Chapman WC, Pinson CW. Positron emission tomography to stage suspected metastatic colorectal carcinoma to the liver. Am J Surg. 1996;171(1):21-6.

13. Trojan J, Schroeder O, Raedle J, Baum RP, Herrmann G, Jacobi V, Zeuzem S. Fluorine-18 FDG positron emission tomography for imaging of Hepatocellular carcinoma. Am J Gastroenterol. 1999;94(11):3314-9.
14. Shiomi S, Nishiguchi S, Ishizu H, Iwata Y, Sasaki N, Tamori A, Habu D, Takeda T, Kubo S, Ochi H. Usefulness of positron emission tomography with fluorine-18-fluorodeoxyglucose for predicting outcome in patients with hepatocellular carcinoma. Am J Gastroenterol. 2001;96(6):1877-80.

15. Kong YH, Han CJ, Lee SD, Sohn WS, Kim MJ, Ki SS, Kim J, Jeong SH, Kim YC, Lee JO, Cheon GJ, Choi CW, Lim SM. Positron emission tomography with fluorine-18-fluorodeoxyglucose is useful for predicting the prognosis of patients with hepatocellular carcinoma. Korean J Hepatol. 2004;10(4):279-87.

16. Goslin R, Steele G Jr, Zamcheck N, Mayer R, MacIntyre J. Factors influencing survival in patients with hepatic metastases from adenocarcinoma of the colon or rectum. Dis Colon Rectum. 1982;25(8):749-54.

17. Steele G Jr, Ravikumar TS. Resection of hepatic metastases from colorectal cancer. Biologic perspective. Ann Surg. 1989;210(2): 127-38.

18. Hustinx R, Paulus P, Jacquet N, Jerusalem G, Bury T, Rigo P. Clinical evaluation of whole-body $18 \mathrm{~F}$-fluorodeoxyglucose positron emission tomography in the detection of liver metastases. Ann Oncol. 1998;9(4):397-401.

19. Arulampalam TH, Francis DL, Visvikis D, Taylor I, Ell PJ. FDGPET for the pre-operative evaluation of colorectal liver metastases. Eur J Surg Oncol. 2004;30(3):286-91.

20. Schiepers C, Penninckx F, De Vadder N, Merckx E, Mortelmans L, Bormans G, Marchal G, Filez L, Aerts R. Contribution of PET in the diagnosis of recurrent colorectal cancer: comparison with conventional imaging. Eur J Surg Oncol. 1995;21(5):517-22.

21. Ogunbiyi OA, Flanagan FL, Dehdashti F, Siegel BA, Trask DD, Birnbaum EH, Fleshman JW, Read TE, Philpott GW, Kodner IJ. Detection of recurrent and metastatic colorectal cancer: comparison of positron emission tomography and computed tomography. Ann Surg Oncol. 1997;4(8):613-20.

22. Fernandez FG, Drebin JA, Linehan DC, Dehdashti F, Siegel BA, Strasberg SM. Five-year survival after resection of hepatic metastases from colorectal cancer in patients screened by positron emission tomography with F-18 fluorodeoxyglucose (FDGPET). Ann Surg. 2004;240(3):438-47.

23. Flanagan FL, Dehdashti F, Ogunbiyi OA, Kodner IJ, Siegel BA. Utility of FDG-PET for investigating unexplained plasma CEA elevation in patients with colorectal cancer. Ann Surg. 1998; 227(3):319-23.

24. Yang M, Martin DR, Karabulut N, Frick MP. Comparison of MR and PET imaging for the evaluation of liver metastases. J Magn Reson Imaging. 2003;17(3):343-9.

25. Kinkel K, Lu Y, Both M, Warren RS, Thoeni RF. Detection of hepatic metastases from cancers of the gastrointestinal tract by using noninvasive imaging methods (US, CT, MR imaging, PET): a meta-analysis. Radiology. 2002;224(3):748-56.

26. Marom EM, McAdams HP, Erasmus JJ, Goodman PC, Culhane DK, Coleman RE, Herndon JE, Patz EF Jr. Staging non-small cell lung cancer with whole-body PET. Radiology. 1999;212(3):803-9.

27. Topal B, Flamen P, Aerts R, D'Hoore A, Filez L, Van Cutsem E, Mortelmans L, Penninckx F. Clinical value of whole-body emission tomography in potentially curable colorectal liver metastases. Eur J Surg Oncol. 2001;27(2):175-9.

28. Ruers TJ, Langenhoff BS, Neeleman N, Jager GJ, Strijk S, Wobbes T, Corstens FH, Oyen WJ. Value of positron emission tomography with [F-18]fluorodeoxyglucose in patients with colorectal liver metastases: a prospective study. J Clin Oncol. 2002;20(2):388-95.

29. Chatziioannou AF. Molecular imaging of small animals with dedicated PET tomographs. Eur J Nucl Med Mol Imaging. 2002;29(1):98-114.

30. Visvikis D, Ell PJ. Impact of technology on the utilisation of positron emission tomography in lymphoma: current and future 
perspectives. Eur J Nucl Med Mol Imaging. 2003;30(Suppl 1): S106-16.

31. Zasadny KR, Wahl RL. Enhanced FDG-PET tumor imaging with correlation-coefficient filtered influx-constant images. J Nucl Med. 1996;37(2):371-4.

32. Rohren EM, Paulson EK, Hagge R, Wong TZ, Killius J, Clavien PA, Nelson RC. The role of F-18 FDG positron emission tomography in preoperative assessment of the liver in patients being considered for curative resection of hepatic metastases from colorectal cancer. Clin Nucl Med. 2002;27(8):550-5.

33. Osman MM, Cohade C, Nakamoto Y, Marshall LT, Leal JP, Wahl RL. Clinically significant inaccurate localization of lesions with PET/CT: frequency in 300 patients. J Nucl Med. 2003;44(2): 240-3.

34. Korin HW, Ehman RL, Riederer SJ, Felmlee JP, Grimm RC. Respiratory kinematics of the upper abdominal organs: a quantitative study. Magn Reson Med. 1992;23(1):172-8.

35. Weiss PH, Baker JM, Potchen EJ. Assessment of hepatic respiratory excursion. J Nucl Med. 1972;13(10):758-9.

36. Nehmeh SA, Erdi YE, Ling CC, Rosenzweig KE, Schoder H, Larson SM, Macapinlac HA, Squire OD, Humm JL. Effect of respiratory gating on quantifying PET images of lung cancer. J Nucl Med. 2002;43(7):876-81.

37. Nakeeb A, Pitt HA, Sohn TA, Coleman J, Abrams RA, Piantadosi S, Hruban RH, Lillemoe KD, Yeo CJ, Cameron JL. Cholangiocarcinoma. A spectrum of intrahepatic, perihilar, and distal tumors. Ann Surg. 1996;224(4):463-73.

38. Pichlmayr R, Weimann A, Klempnauer J, Oldhafer KJ, Maschek $\mathrm{H}$, Tusch $\mathrm{G}$, Ringe B. Surgical treatment in proximal bile duct cancer. A single-center experience. Ann Surg. 1996;224(5): 628-38.

39. Zimmerman RL, Fogt F, Burke M, Murakata LA. Assessment of Glut-1 expression in cholangiocarcinoma, benign biliary lesions and hepatocellular carcinoma. Oncol Rep. 2002;9(4):689-92.

40. Kato T, Tsukamoto E, Kuge Y, Katoh C, Nambu T, Nobuta A, Kondo S, Asaka M, Tamaki N. Clinical role of (18)F-FDG PET for initial staging of patients with extrahepatic bile duct cancer. Eur J Nucl Med Mol Imaging. 2002;29(8):1047-54.

41. Kim YJ, Yun M, Lee WJ, Kim KS, Lee JD. Usefulness of 18FFDG PET in intrahepatic cholangiocarcinoma. Eur J Nucl Med Mol Imaging. 2003;30(11):1467-72.

42. Fritscher-Ravens A, Bohuslavizki KH, Broering DC, Jenicke L, Schafer H, Buchert R, Rogiers X, Clausen M. FDG PET in the diagnosis of hilar cholangiocarcinoma. Nucl Med Commun. 2001;22(12):1277-85.

43. Bartolozzi C, Cioni D, Donati F, Lencioni R. Focal liver lesions: MR imaging-pathologic correlation. Eur Radiol. 2001;11(8): 1374-88.

44. McGhana JP, Dodd GD 3rd. Radiofrequency ablation of the liver: current status. Am J Roentgenol. 2001;176(1):3-16.

45. Livraghi T, Goldberg SN, Lazzaroni S, Meloni F, Ierace T, Solbiati L, Gazelle GS. Hepatocellular carcinoma: radio-frequency ablation of medium and large lesions. Radiology. 2001; 218(3):918-9.

46. Donckier V, Van Laethem JL, Goldman S, Van Gansbeke D, Feron P, Ickx B, Wikler D, Gelin M. [F-18] fluorodeoxyglucose positron emission tomography as a tool for early recognition of incomplete tumor destruction after radiofrequency ablation for liver metastases. J Surg Oncol. 2003;84(4):215-23.
47. Strumberg D, Richly H, Hilger RA, et al. Phase I clinical and pharmacokinetic study of the novel Raf kinase and vascular endothelial growth factor receptor inhibitor BAY 43-9006 in patients with advanced refractory solid tumors. J Clin Oncol. 2005;23:965-72.

48. Llovet JM, Ricci S, Mazzaferro V, Hilgard P, Gane E, Blanc JF, de Oliveira AC, Santoro A, Raoul JL, Forner A, Schwartz M, Porta C, Zeuzem S, Bolondi L, Greten TF, Galle PR, Seitz JF, Borbath I, Häussinger D, Giannaris T, Shan M, Moscovici M, Voliotis D, Bruix J; SHARP Investigators Study Group. Sorafenib in advanced hepatocellular carcinoma. $\mathrm{N}$ Engl $\mathrm{J}$ Med. 2008;359(4):378-90.

49. Siemerink EJ, Mulder NH, Brouwers AH, Hospers GA. 18F Fluorodeoxyglucose positron emission tomography for monitoring response to sorafenib treatment in patients with hepatocellular carcinoma. Oncologist. 2008;13(6):734-5.

50. Lee JH, Park JY, Kim do Y, Ahn SH, Han KH, Seo HJ, Lee JD, Choi HJ. Prognostic value of 18 F-FDG PET for hepatocellular carcinoma patients treated with sorafenib. Liver Int. 2011;31(8): 1144-9.

51. De Bruyne S, Van Damme N, Smeets P, Ferdinande L, Ceelen W, Mertens J, Van de Wiele C, Troisi R, Libbrecht L, Laurent S, Geboes K, Peeters M. Value of DCE-MRI and FDG-PET/CT in the prediction of response to preoperative chemotherapy with bevacizumab for colorectal liver metastases. Br J Cancer. 2012; 106(12):1926-33.

52. Bertolini F, Malavasi N, Scarabelli L, Fiocchi F, Bagni B, Del Giovane C, Colucci G, Gerunda GE, Depenni R, Zironi S, Fontana A, Pettorelli E, Luppi G, Conte PF. FOLFOX6 and bevacizumab in non-optimally resectable liver metastases from colorectal cancer. Br J Cancer. 2011;104(7):1079-84.

53. Seo S, Hatano E, Higashi T, Hara T, Tada M, Tamaki N, Iwaisako K, Ikai I, Uemoto S. Fluorine-18 fluorodeoxyglucose positron emission tomography predicts tumor differentiation, P-glycoprotein expression, and outcome after resection in hepatocellular carcinoma. Clin Cancer Res. 2007;15(13):427-33.

54. Ho CL, Chen S, Yeung DW, Cheng TK. Dual-tracer PET/CT imaging in evaluation of metastatic hepatocellular carcinoma. J Nucl Med. 2007;48:902-9.

55. Delbeke D, Pinson CW. 11C-acetate: a new tracer for the evaluation of hepatocellular carcinoma. J Nucl Med. 2003;44:222-3.

56. Sugiyama M, Sakahara H, Torizuka T, Kanno T, Nakamura F, Futatsubashi M, Nakamura S. 18F-FDG PET in the detection of extrahepatic metastases from hepatocellular carcinoma. J Gastroenterol. 2004;39(10):961-8.

57. Lee JE, Jang JY, Jeong SW, Lee SH, Kim SG, Cha SW, Kim YS, Cho YD, Kim HS, Kim BS, Jin SY, Choi DL. Diagnostic value for extrahepatic metastases of hepatocellular carcinoma in positron emission tomography/computed tomography scan. World $\mathbf{J}$ Gastroenterol. 2012;18(23):2979-87.

58. Kim JY, Kim MH, Lee TY, et al. Clinical role of 18F-FDG PET$\mathrm{CT}$ in suspected and potentially operable cholangiocarcinoma: a prospective study compared with conventional imaging. Am J Gastroenterol. 2008;103:1145-51.

59. Jadvar H, Henderson RW, Conti PS. [F-18]fluorodeoxyglucose positron emission tomography and positron emission tomography: computed tomography in recurrent and metastatic cholangiocarcinoma. J Comput Assist Tomogr. 2007;31:223-8. 